\title{
Vapour Deposition of Gold Alloys
}

\author{
DEVELOPMENTS IN RADIO-FREQUENCY SPUTTERING \\ OF ELECTRICAL CONTACT SURFACES
}

\author{
Walter Reichelt \\ W. C. Heraeus GmbH, Hanau, Federal German Republic
}

The vacuum deposition processes of thermal evaporation and cathodic sputtering have for many years made it possible to produce thin films of gold on any other metal. The successful production of gold alloy coatings of controlled composition now offers a much wider choice of materials to the designer of light duty electrical contacts.

The trend towards miniaturisation in electronics, especially in the telecommunications industry, involves the use of both moving contacts, as in reed relays, and interconnections which have much thinner contact coatings than are used in larger scale components. At the same time, it is essential to maintain the utmost reliability in operation. Contamination by unwanted metals tends to increase the contact resistance beyond acceptable limits, for example in low contact pressure switches controlling small currents. The presence of very thin contaminating layers also increases the contact resistance of static connectors of the "plug-in" type. Similarly, contamination impairs the joining of active components by soldering, welding or eutectic bonding.

It is almost impossible to avoid some contamination when coating is carried out by rolling or by electroplating. By contrast, contamination is avoided by employing vacuum evaporation, sputtering or chemical vapour deposition since the coating is deposited under extremely clean conditions. Chemical vapour deposition generally requires high substrate temperatures in the range 800 to $1200^{\circ} \mathrm{C}$ and cannot be used to deposit gold or gold alloys on work-hardened spring materials.

Vacuum evaporation, using electron-beam heating, produces excellent fine gold films but is less suitable for gold alloys, as the rate of evaporation of each constituent of the alloy is different. The process can be adapted for depositing alloys by evaporating each constituent from a separate crucible. Because it is difficult to control several electron beams to maintain the correct alloy constitution, a single electron beam is used which is rapidly directed to each crucible in turn, using electronically controlled electromagnetic deflector plates. The correct alloy constitution is maintained by monitoring the vapour pressure of each constituent using, for example, atomic absorption. Another method of depositing binary alloys is to feed the component having the higher vapour pressure continuously to a molten pool of the lower vapour pressure component. Finally, there is the relatively old method for the vacuum deposition of alloys known as flash evaporation. The alloy is prepared as a fine wire or powder and is slowly fed or dropped on to an evaporation surface held at a sufficiently high temperature to evaporate all the components instantaneously. The method has its disadvantages; it is difficult to avoid spitting, and for many metals there is no crucible material which does not react at the very high temperatures required. Furthermore, the evaporated coating may not have the same structure as the starting material even though its composition as a whole is correct. The individual components are deposited as single atoms and have to rearrange on the surface to form the required alloy. This is not a fundamental difficulty, however, as the energy to produce the necessary rearrangement is partly provided by the liberated latent heat of condensation and can be augmented by heating the substrate during the deposition. In some cases it is possible to form an alloy coating by subsequently heating two or more homogeneous films which have been deposited successively on the substrate. This can be done, for example, with separate layers of gold and silver.

The most elegant and least complicated method for depositing films of alloys is radio-frequency sputtering. This is a special version of the wellknown cathode sputtering process in which atoms of the metallic cathode are vaporised by bombardment with positively charged rare gas ions. The sputtered atoms then condense on the surface of the substrate placed a short distance away. As in flash evaporation, small amounts of the target material are continuously carried over in the correct proportions. The removal 


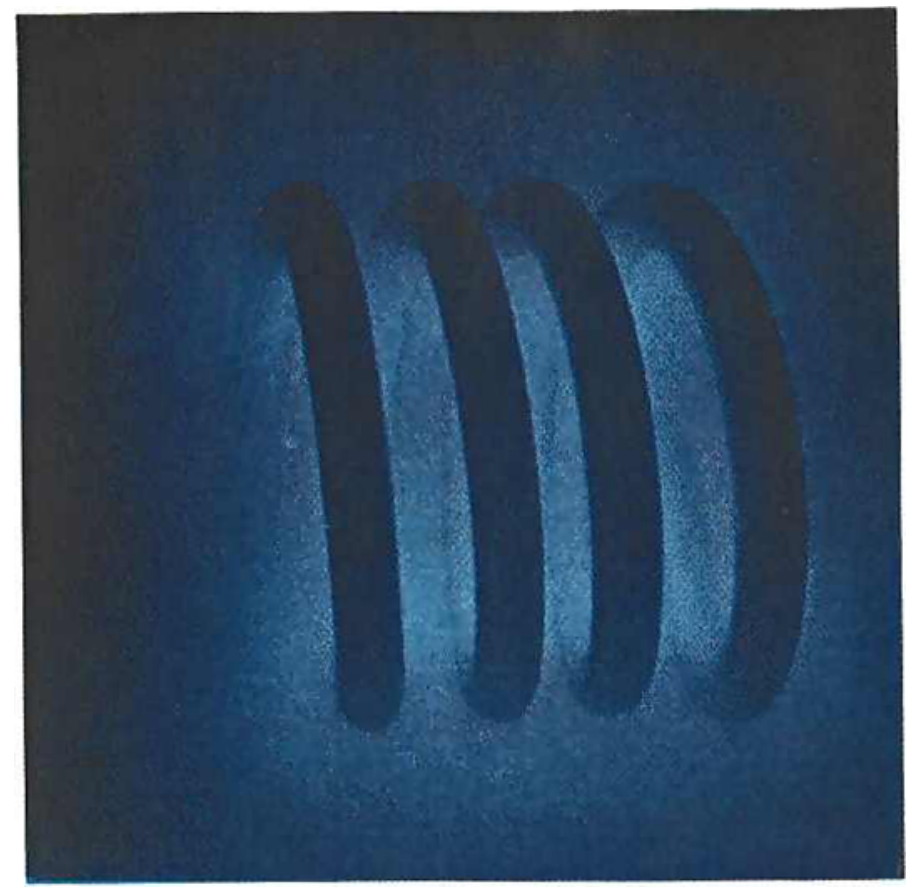

Fig. I In the production of films of gold alloys of controlled composition by high-frequency sputtering, a water-cooled copper coil, clad with the gold alloy to be deposited, is bombarded with positively charged argon ions, the sputtered atoms then condensing on the surface of the substrate in the correet proportions. One arrangement is a cylindrical coil such as this. The discharge can clearly be seen, and is confined mainly to the inner volume. For deposition on plane surfaces a flat rectangular or circular coil can be used

of target material, however, does not occur as a result of thermal lattice vibrations but by the transfer of momentum from the bombarding ions. The kinetic energy of the atoms removed from the target is four to five times greater than that of atoms released by thermal evaporation. This increased kinetic energy is liberated during condensation and facilitates the formation of the alloy structure in the film. The sputtering rate is greater when the target surface is very pure, especially when it is free from oxides, nitrides, carbides, etc. This requirement is naturally fulfilled by the noble metals, which have the highest sputtering rate of all. Apart from surface purity, the sputtering rate depends on the ion density

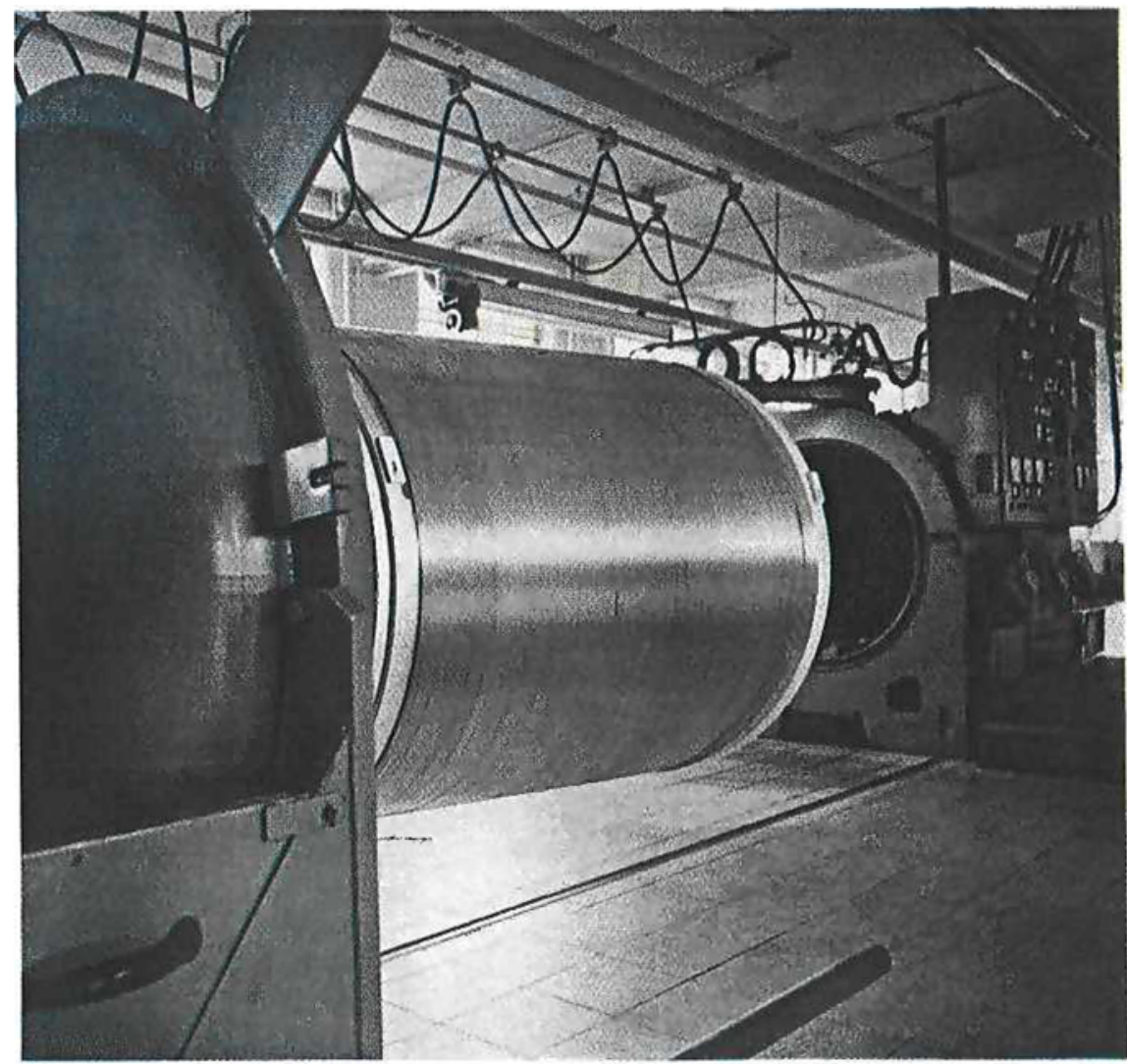

Fig. 2 A high vacuum production plant for the de. position of gold alloys on to flat spring strip. Coating can be confined to the limited contact areas of the strip 
Fig. 3 A typical strip of nickel-silver coated with two stripes of a gold-silvercopper alloy several microns thick. This material can then be stamped and formed into contact springs

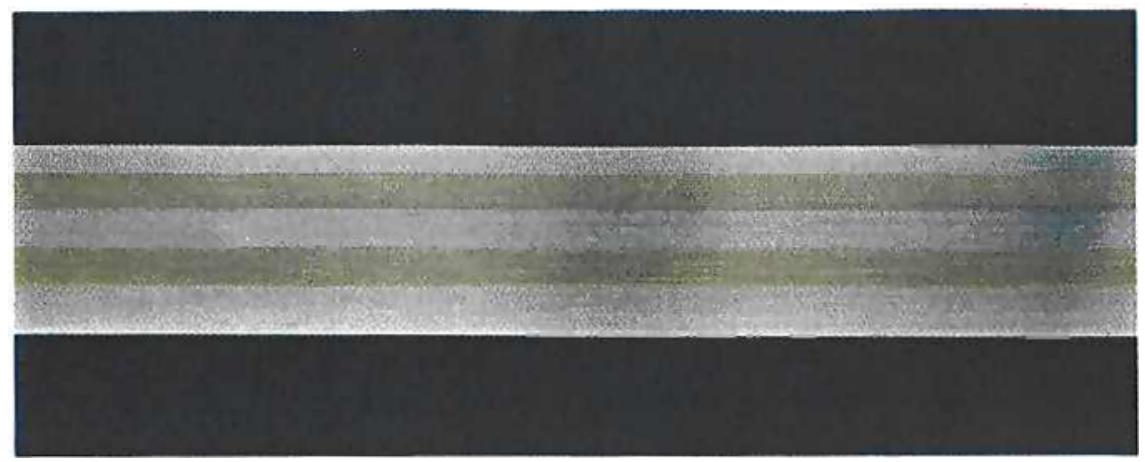

and on the number of target atoms which are removed by a single ion impact (the specific sputtering rate). It is only possible to increase the ion density to a certain extent when carrying out direct current sputtering using a self-sustained discharge. As soon as a limiting current of a few amperes is exceeded, the discharge transforms to an uncontrollable arc which wanders about the target surface in a random manner. It is, therefore, more satisfactory to use methods other than increasing the gas pressure to raise the ion density, such as the use of an auxiliary thermionic cathode or the application of a radiofrequency alternating current discharge.

The high frequency method is preferable as it is more versatile and allows of higher energy densities in the discharge. In principle, ionised plasma can be produced by an electrodeless circular discharge, the induction coil remaining outside the glass or ceramic vacuum chamber. An interesting variation of the technique is to fabricate the plasma generating coil from the target metal and place it inside the vacuum chamber (German Patent 1,515,296.1). An energy output of $20 \mathrm{~kW}$ can be readily obtained in a volume of 0.3 litre using a cylindrical coil, or over an area of $200 \mathrm{~cm}^{2}$ using a rectangular or circular flat coil. The bombardment resulting from such high power inputs generates a high temperature in the coil and can easily lead to melting. It is, therefore, necessary to provide efficient water cooling, for example, a water-cooled copper tube covered with the alloy to be sputtered. It is essential to ensure good thermal contact between the copper and the alloy.

Efficient cooling is also necessary to prevent thermal evaporation being superimposed on the sputtering process as this would lead to fractionation of the components. It has been observed that in the case of a pale yellow gold-silver-copper alloy those areas which are insufficiently cooled tend to assume a more reddish colour after sputtering because silver (the component with the highest vapour pressure) is the first metal to evaporate leaving the alloy with a smaller silver content in the overheated areas. If the cooling is adequate, the deposited film will have the same composition as the original alloy. This fact can be checked by X-ray fluorescence analysis. As a general rule, it is advisable to use vacuum melted alloys as starting materials in order to avoid spitting caused by dissolved gases.

Figure 1 shows a cylindrical coil used for the plasma discharge. With high energy inputs, the colour of the discharge is characteristic of the metal of the coil. The discharge can be maintained at pressures down to $10^{-4}$ torr. Sputtering rates of $1 \mu \mathrm{m}$ per minute can be achieved without difficulty.

Figure 2 shows a production plant for coating flat spring strip. The range of alloys which have been tested include gold-palladium, gold-nickel, gold-silver, and gold-silver-copper, all of which form solid solutions. It is also possible to produce heterogeneous mixtures such as gold and molybdenum or gold and tungsten which are of interest for certain types of switchgear contacts.

Practical tests have shown that the physical and chemical properties of the alloy coatings come up to expectation in respect of hardness, abrasion resistance, and conductivity.

\section{Gold Plating in Computer Memory Elements}

Wires having an electrodeposited film of nickeliron alloy form the essential feature of computer memory elements, but their magnetic properties are critically dependent on the structure and surface finish of the underlying copper deposit. Their performance has now been found to be improved by the electrodeposition of gold between the copper and the nickeliron layers. During an investigation by F. E. Luborsky, M. W. Breiter and B. J. Drummond of General Electric Corporate Research and Development ( $\mathcal{F}$. Electrochem. Soc., 1972, 119, (1), 92) the deposition of gold at very low current densities on to a rough and coarsegrained copper deposit produced islands of gold by selective deposition on to the high points of the copper surface, and performance was found to be related to a specific structure of these islands. 